\title{
Procesos de jerarquización en secundarias universitarias en Argentina
}

\author{
Hierarchical processes in university \\ secondary schools in Argentina
}

\author{
María Emilia Di Piero \\ Consejo Nacional de Investigaciones Científicas y Técnicas/Argentina \\ Facultad Latinoamericana de Ciencias Sociales/Argentina \\ Universidad Nacional de La Plata \\ medipiero@gmail.com \\ Código Orcid: http://orcid.org/0000-0002-1500-7542
}

\begin{abstract}
Resumen
Se analizan procesos de legitimación de desigualdades socioeducativas en escuelas secundarias enfocándose en la producción de asimetrías a partir de la afirmación de distancias con respecto a "los otros" en prestigiosas instituciones dependientes de una Universidad Nacional en Argentina. El enfoque metodológico partió de un estudio de caso múltiple. Entre 2012 y 2016 se realizaron observaciones, análisis documental, 13 entrevistas en profundidad a actores "clave" y 67 semiestructuradas a docentes. Los resultados muestran procesos múltiples y superpuestos de "autolegitimación por distanciamiento": los "otros" son las escuelas dependientes de la Provincia de Buenos Aires, las privadas, las otras de pregrado o la otra especialidad. Las fronteras son móviles: un trabajo de jerarquización consolida a estas escuelas en un lugar de elite.
\end{abstract}

\section{Palabras clave}

Escuela secundaria, desigualdad social, elite, educación, diferenciación cultural, fronteras.

\section{Abstract}

This paper analyzes processes of legitimization of socio-educational inequalities in secondary schools focusing on the production of asymmetries based on the affirmation of distances with respect to "the others" in prestigious institutions dependent on a National University in Argentina. The methodological approach was based on a multiple case study. Between 2012 and 2016 were made observations, documentary analysis, 13 in-depth interviews with "key" actors and 67 semi-structured interviews with teachers. The results show multiple and overlapping processes of "self-legitimization by distancing": the "others" are the dependent schools of the Province of Buenos Aires, the private schools, the other undergraduate schools or the other specialty. Borders are mobile: a work of hierarchy consolidates these schools into an elite place.

\section{Keywords}

Secondary schools, social inequality, elite, cultural differentiation, boundaries.

Forma sugerida de citar: Di Piero, María Emilia (2018). Procesos de jerarquización en secundarias universitarias en Argentina. Universitas, 28, pp. 61-78. 


\section{Introducción y estado de la cuestión}

En este trabajo se analizan procesos de legitimación de desigualdades socioeducativas en escuelas secundarias poniendo el foco en la producción de jerarquías a partir de fronteras simbólicas con respecto a "los otros" presentes en un grupo prestigioso y sobredemandado ${ }^{1}$ de instituciones dependientes de una Universidad Nacional en Argentina. Desde un enfoque relacional de las desigualdades, se estudian procesos de autolegitimación por distanciamiento que permiten a este grupo de escuelas ubicadas en la ciudad de La Plata, capital de la Provincia de Buenos Aires, distinguirse mediante la subalternización de sus "otros" y constituirse como elites a partir de la delimitación de fronteras.

Como es sabido, la construcción de circuitos educativos desiguales no se subsume a la existencia de escuelas públicas y privadas sino que las desigualdades se multiplican y superponen. De este modo, existen escuelas privadas que atienden a sectores populares y otras a sectores medios y altos. La misma dinámica se replica en el sector público, como se observa en las escuelas seleccionadas en esta investigación.

Se trata de un grupo de instituciones estatales que históricamente atendió a sectores medios y altos reclutados mediante examen de ingreso eliminatorio y que, en los años 80 -en el contexto de apertura democrática tras la última y más feroz dictadura cívico militar en el país- modificaron su método de admisión hacia el sorteo público, adaptándose a la configuración que adquiría el mandato "democratizador" en ese momento. Una de las escuelas, de especialidad artística, adoptó parcialmente el sorteo en los años 80 y lo hizo completamente sólo a partir de 2015, cuando el significante "inclusión" se convirtió en palabra clave del clima epocal. Cabe agregar que la Universidad Nacional de La Plata cuenta con tres niveles: pregrado, grado y posgrado. Al primero de ellos pertenecen cinco establecimientos, cuatro de educación secundaria y uno de primaria: un secundario con orientación agrotécnica se ubica en el partido de 25 de Mayo (Provincia de Buenos Aires) y otros cuatro en la Ciudad de La Plata. Tres de estos últimos son escuelas secundarias, que en el presente trabajo serán identificadas como Escuela 1,2 y 3.

1 En el año 2017 fueron 687 los inscriptos para 184 vacantes en la Escuela 3539 inscriptos para 130 vacantes en la Escuela 1 y 370 aspirantes para 85 vacantes en el caso de la Escuela 2 según información oficial. 
Como ha indicado Reygadas (2008), numerosos trabajos abordan la idea de que a partir de clasificaciones las sociedades establecen límites que definen conjuntos de relaciones a partir de los cuales se crea determinado orden jerárquico. Si bien hay diferencias entre distintos autores que han referido a esta problemática, se rescata el poder jerarquizador de las clasificaciones sociales. Por su parte, Max Weber (1969) postulaba la existencia de grupos de status explicados por la distribución desigual del prestigio social. Asimismo, Gerard Cohen (1996) refiere a la "mística de la excelencia" y a los "cultos de elite" que permiten a un grupo validar y sostener su estatus privilegiado al afirmar que poseen cualidades escasas y exclusivas que son esenciales para la sociedad en su conjunto.

El análisis de Charles Tilly (2000) sobre los pares categoriales también nos muestra que el plano simbólico colabora en la construcción de límites que actúan como fronteras entre grupos sociales. Este autor responde a un enfoque relacional de la desigualdad (Stich y Colyar, 2013), estudiando el trabajo de construcción y reproducción de límites simbólicos (boundary work). Para el autor las categorías dicotómicas constituyen estrategias de los grupos dominantes en pos de excluir a otros grupos y bloquear el acceso a determinados recursos.

Por su parte, Norbert Elías (1998) estudia una comunidad urbana, a la que llama Winston Parva, caracterizada por la división entre dos grupos de residentes: un grupo obrero establecido hace más tiempo en relación con un nuevo asentamiento de obreros. El autor señala que en esa pequeña comunidad se observa un tema universal en miniatura: "en todos esos casos el grupo más poderoso se ve a sí mismo como gente mejor" (1998, p. 82). Entre los residentes no había diferencias en cuanto a nivel educativo, ocupación, ingresos, etnia, sin embargo, los "recién llegados" eran estigmatizados como personas de valor humano inferior. En ese sentido, señala que cualquier teoría que explique los diferenciales de poder exclusivamente en relación con la posesión monopólica de objetos resulta limitada, por el contrario, él explica procesos de distinción que se generan a partir de las diferencias de antigüedad. A continuación se analizan procesos de fabricación de asimetrías presentes en las escuelas abordadas en las cuales, como se mostrará, las jerarquizaciones se yuxtaponen y las fronteras son inestables. 


\section{Material y métodos}

El enfoque metodológico partió de un estudio de caso múltiple que combinó metodologías cualitativas para la recolección de datos. El estudio de caso se centra en las dinámicas que acontecen en escenarios particulares (Eisenhardt, 1989): en esta investigación, dos escuelas de pregrado dependientes de la Universidad Nacional de La Plata ubicadas en la ciudad homónima. Una de ellas adoptó completamente el mecanismo de sorteo como forma de ingreso desde mediados de los años 80 basándose en un discurso "democratizador". La otra adoptó ese mecanismo de admisión parcialmente en el momento de retorno de la democracia al país, incorporándolo para el total de aspirantes sólo a partir del año 2015 en un contexto en que gravitó una narrativa en favor de la "inclusión" educativa.

Siguiendo la clasificación propuesta por Stake (1995), esta investigación parte del tipo de estudio de caso "instrumental" en tanto el interés no reside sólo en el caso en sí mismo sino que la moviliza un problema conceptual más amplio que el estudio de estos casos particulares puede iluminar. En ese sentido, se exploran procesos de producción y legitimación de desigualdades socioeducativas poniendo el foco en la construcción de jerarquías a través del estudio de dos de las tres instituciones medias dependientes de una Universidad Nacional situadas en la capital de la Provincia de Buenos Aires.

Entre los años 2012 y 2016 fueron realizadas observaciones en ambas escuelas, entrevistados en profundidad trece actores "clave" para las instituciones (directivos, jefes de departamentos, secretarios de extensión y secretarios académicos de las escuelas y de la universidad), efectuadas sesenta y siete entrevistas semiestructuradas con preguntas abiertas y cerradas a docentes seleccionados de modo aleatorio ${ }^{2}$, recuperado un corpus compuesto por seis documentos institucionales que incluyeron información sociodemográfica de los/as ingresantes y sobre los métodos de admisión vigentes en las secundarias dependientes de universidades nacionales. A continuación analizaremos los principales resultados de la investigación en lo que refiere a la

2 Según Stake (1995), existen varias posibilidades para seleccionar los componentes en una muestra probabilística. En la presente investigación optamos por una muestra sistemática: se tomó el listado de docentes de cada escuela y a partir de un punto de arranque se realizó el conteo considerando formaciones disciplinares diversas. 
construcción de fronteras que posicionan a estas instituciones en un lugar de elite desde las miradas de los docentes y actores clave.

\section{Ni provincial ni privado: universitario. Fronteras simbólicas entre escuelas universitarias, provinciales y privadas}

Como somos pocos y nos conocemos mucho en esta ciudad chica, encuadrada entre avenidas y cruzada por sus diagonales, [la Escuela 1] ha dispuesto el seguimiento de sus alumnos por las aulas y perdidos corredores de las facultades (...) Y con mucho asombro ha confirmado (...) lo que todos sabemos: el bachiller de la escuela 1 se adapta a cualquier circunstancia, resuelve con solvencia los problemas y se destaca entre sus pares. Una pinturita. (...) En sus 53 años de colegio de señoritas y sus 40 de [Escuela 1], colegio mixto, se puede ufanar de haber sido un semillero intelectual, un vivero de peces coloridos; porque entre la fauna y la flora de sus egresados brillan los galardones, los becarios, los investigadores exitosos y los descubridores a nivel internacional, sin retaceos (UNLP, 2001, p. 46).

Las fronteras simbólicas son múltiples y se superponen. Retomando los aportes de Amy Siych y Julia Colyar (2013), lejos de todo binarismo, no puede pensarse que las distancias estén fijas de una vez y para siempre, sino que se trata de límites móviles que demarcan los grupos en cierto momento y en oposición a un "otro" determinado. Es decir que no existe un "nosotros" fijo y homogéneo sino que la figura de "el otro" es porosa e inestable en tanto se delimita en función de la producción del propio grupo (Latour, 2008) y puede ser, incluso, parte del nosotros en un tiempo y espacio dados.

Es posible analizar el esfuerzo de docentes y actores clave consultados en las Escuelas 1 y 2 en pos de diferenciarse de aquellos a quienes instituyen como sus "otros" en la clave de que todas las elites realizan un trabajo para constituirse como tales. Dicho trabajo resulta necesario para performar asimetrías que los afirman en su lugar; el "otro" en este caso está constituido por tres frentes: por un lado, por las escuelas privadas; por otro, por las escuelas "de provincia"; por último, por las otras dos escuelas que conforman el grupo de establecimientos secundarios de pregrado en la Ciudad de La Plata.

Con respecto a la diferenciación en el caso de las escuelas privadas, aflora una dimensión ideológica cuando se subraya que la educación no es un servi- 
cio a ser "mercantilizado": los entrevistados sostienen que no "gastarían" en educación, puesto que es deber del Estado asegurarla. Así, para el caso de la Escuela 1 la directora electa, a quien llamaremos Juliana, indicaba:

Es más ideológico, yo no mandaría a una escuela privada de ningún tipo porque me parece que la escuela es pública y que el plus que da la escuela pública... aunque en privada tengan el mejor aire acondicionado, el último plasma, último modelo con toda la tecnología digital para ver... hay cosas que no te da el gueto de la escuela privada...(Juliana, directora, Escuela 1).

En estos discursos los entrevistados aparecen, como indicaban Crozier et al. (2008) al analizar la elección escolar de la clase media blanca en el Reino Unido, como los “campeones de la educación pública”. Se trata, sin embargo, de un tipo particular de educación pública en el que no se opta por escuelas privadas siempre y cuando se mantenga la posibilidad de la sociabilidad entre familias de clase media en escuelas públicas. Crozier refiere a padres que monitorean la situación desde lo que denomina "una ambigüedad moral de clase media" en tanto pretenden, por un lado, ser igualitarios y, por otro, mejorar su posición social.

En los años 90, en un contexto de deterioro de las instituciones educativas estatales en el país en el que las mismas se encontraron subdotadas de recursos y sobrecargadas de funciones (Tenti Fanfani, 2011, p. 146), se produjo el abandono masivo de este tipo de establecimientos por parte de sectores de mayor poder adquisitivo, impulsando el boom de los establecimientos privados. Si bien la expansión del circuito privado había comenzado en los años 60 en un proceso de diferenciación que acompañaba al contexto de ampliación de la escolarización, en los años 90 la lógica del mercado ganó espacio: las escuelas se transformaron en mercancías adquiridas según la capacidad de consumo. Precisamente de esta lógica buscan diferenciarse en sus discursos las/os entrevistadas/os en estas escuelas.

Asimismo, estas narrativas que se pronuncian contra la educación privada remiten a los señalamientos de Walzer (1993). En su análisis de las esferas de la justicia, sostiene que la distribución desigual de bienes dentro de una misma esfera no tiene por qué ser objetable, sino que la injusticia surge cuando los bienes se convierten en otros bienes, mediante una transgresión de esferas. En la concepción de Walzer, unos pueden ser más ricos que otros, el problema es cuando esa fortuna les sirve para obtener, por ejemplo, una mejor educación. 
También la Prosecretaria Académica de la Universidad Nacional, a quien llamaremos Liliana, se refería a este punto. Al diferenciar a las escuelas de pregrado de las escuelas privadas, señalaba:

La educación pública tiene para mí esta virtud de que recibe lo que el Estado decide en su conjunto como educación para todo el país, y la escuela es el Estado y vive dentro del Estado, como ciudadano, y eso es algo bueno. No hay ninguna idea que esté direccionando la formación del alumno: no hay una sola idea. Uno podría encontrarse con escuelas privadas que no son religiosas pero que plasman un paradigma de enseñanza. (...) en cambio una escuela plural te prepara para la vida (Liliana, Prosecretaria Académica UNLP).

Así, destaca la pluralidad como uno de los rasgos específicos que diferencias estas escuelas de la oferta privada en donde existe, en su visión, un sentido monolítico otorgado a la educación. Sin embargo, también en estas escuelas se encontró una definición única del tipo de "estudiante autónoma/o" esperado y del tipo de subjetivación "crítica" a habilitar compartida unánimemente por los actores consultados.

Del mismo modo, Liliana indicaba una diferencia material dada por la desigualdad de recursos:

Contamos con infraestructura, contamos con cargos, esa es una gran diferencia con la privada. Porque mi hijo terminó la primaria en una escuela privada laica y no había biblioteca. Y es una escuela privada muy buena. Y, cuando mi hijo se iba, empezaron a armar una biblioteca... no hay DOE [Departamento de Orientación Escolar]. Era contenidista, clásica. Yo lo tuve que sacar del normal X. Yo fui al normal XX, pero a él lo tuve que sacar. La maestra me manda una nota en un cuaderno y [la maestra] tenía 9 faltas de ortografía (...) Entonces al chico en cuarto grado lo saqué. Entonces yo te digo: [en las escuelas de la universidad] hay servicios, oferta, personal, que no está ni en el privado ni en la provincia (Liliana, Prosecretaria Académica UNLP).

En ese sentido, la mayor disponibilidad de recursos con que cuentan las escuelas de pregrado emerge como otra de las características que las diferencian de las escuelas privadas. Asimismo, resulta interesante detenerse, dentro del argumento de Liliana, en la encrucijada entre los valores republicanos e igualitarios y los intereses en torno a la educación de su propio hijo. 
En el caso de las estrategias de autoproducción y de diferenciación en relación con las escuelas provinciales, los entrevistados subrayaban ciertos rasgos de las escuelas de pregrado que operan como factores de distinción.

Una primera característica distintiva del grupo de escuelas de pregrado según sus docentes es que el ciclo lectivo comienza después y termina antes, pero es, en sus términos, de "mejor calidad" en comparación con el de las escuelas provinciales. En ese sentido, una docente de Ciencias Naturales indicaba:

[el estudiantado] Es heterogéneo pero los que egresan son siempre los mismos. Esto no es la provincia de Buenos Aires, acá el chico se tiene que esforzar, y a veces terminan pensando: "si acá me exigen, mejor me voy a la de provincia que no me exigen" (Docente $n^{\circ} 22$; Escuela 1 ).

De este modo, estos discursos construyen a estas escuelas como más demandantes de esfuerzo por parte de las/os estudiantes en comparación con las provinciales. Por su parte, Juliana sostenía:

Tengo dos alumnas, sus padres son bolivianos, y vienen de la periferia: eran muy buenas alumnas en sus escuelas, acá les va mal... y además todo el tema de cómo eso pega en la autoestima. Y esas son sus maestras, las que les hicieron conocer la posibilidad de esta escuela... (Juliana, directora, escuela 1).

Ciertamente, su preocupación en relación con que a algunas estudiantes que presentaban los mejores promedios en una escuela provincial, en la Escuela 1 "les va mal" se relaciona con la tesis que señala la fragmentación (Kessler, 2002; Tiramonti, 2004; Núñez y Litichever, 2015) existente entre establecimientos educativos que se consolidan como mundos inconmensurables y autocentrados. Como muestra Saraví (2015), el sistema educativo tiende a un proceso de exclusiones recíprocas e inclusiones desiguales que supone que se transiten experiencias sólo vinculadas a lo conocido y entre sectores sociales similares.

También desde la Escuela 2, una docente indicaba: "Porque son escuelas que están por encima de las demás, el nivel es más alto. Es necesario que quienes ingresan se ganen el lugar" (Docente $n^{\circ} 18$, escuela 2 ).

Retomando a Oscar Aguilar, las elites se encuentran en todo ámbito en el que resulte posible jerarquizar a los individuos en virtud de ciertos criterios, y dicha jerarquización se sustenta en una autocomprensión de las pro- 
pias elites como superiores (2011, p. 205). En este caso, los miembros de las escuelas universitarias se consideran superiores a un conjunto externo $a$ priori homogéneo.

Así, en el imaginario educativo de los entrevistados las escuelas de pregrado emergen como islas que promueven una escolarización de alta intensidad en comparación con "el resto" de las escuelas públicas. También la Secretaría de Extensión de la Escuela 2 se refería a la calidad educativa que las familias procuran en esta Escuela:

Me parece que los colegios de la universidad también es, dentro de lo público, una currícula, una manera de educarte que todavía tiene un nivel, el nivel de formación de las personas que trabajan ahí...yo también trabajo en la facultad, y ahí está esa mirada de "si me animo a trabajar en secundario, no voy a trabajar en cualquier escuela, voy a trabajar en los colegios de la universidad". Es esa garantía de que hay un andamiaje en los colegios de la universidad (Nina, Secretaria de Extensión, Escuela 2).

De ese modo, se considera que la sobredemanda de estas escuelas guarda relación con que ocupan un lugar de superioridad en el imaginario educativo platense: en la elección de estos establecimientos se juega el "elegir una buena escuela" para las/os hijas/os. Una segunda característica distintiva de este grupo de escuelas es que cuentan con un presupuesto mayor en comparación con las provinciales. La directora electa en la Escuela 1 indicaba al respecto:

Es cierto que tenemos más plata que el resto: eso es una realidad material objetiva, sobre todo en este último tiempo hay más dinero. Entonces eso te permite hacer más cosas, hasta te permite estar mejor estéticamente. Mi hijo, yo tengo mellizos... uno va a la Escuela 3, a primer año, y otro va al normal $\mathrm{X}$. Vos entrás al normal y te da ganas de sacarlo: las ventanas no tienen vidrio, las puertas no tienen picaporte, sus pizarroncitos...acá la escuela está pintada, el baño funciona, tienen papel higiénico, tienen pizarrón nuevo, tienen fibrón (Juliana, directora, escuela 1).

Por su parte, la Prosecretaria de Asuntos Académicos de la Universidad señalaba al respecto:

Hay una diferencia básica entre nosotros y la provincia de Buenos Aires que es la infraestructura... la anterior directora de secundaria [de la provincia] siempre nos decía: "necesito un piso de presupuesto que no tengo". Entonces 
eso ya te hace una diferencia fundamental... nosotros no tenemos problemas de ambiente, no tenemos problemas de recursos, no tenemos problemas de servicios de internet, no tenemos problemas de conectar igualdad, ¡no tenemos problemas! Tenemos becas de transporte, de apuntes, alimentaria, cargos, ¿qué problema tenemos? Tenemos personal de apoyo, tenemos departamento de orientación. ¿Por qué tenemos deserción? Y no lo puedo soportar, yo no puedo soportar que no todos terminen la escuela, que terminen con bancos vacíos (Liliana, Prosecretaria de Asuntos Académicos UNLP).

La Prosecretaria indicaba su preocupación frente a la deserción, dado que, a diferencia de la situación en provincia, en las escuelas de pregrado se cuenta con los recursos para impedirla.

Un tercer rasgo particular de las escuelas de pregrado refiere al Régimen Académico, específicamente en cuanto a la repitencia: a partir del año 2010 , los estudiantes pueden repetir sólo una vez, debiendo luego cambiarse de escuela, restricción inexistente en las escuelas provinciales. De ese modo, las escuelas universitarias se resguardan como guetos de calidad, en tanto se aseguran la expulsión de los estudiantes repetidores. En el mismo sentido, también desde el año 2010, la repitencia de los estudiantes se produce al adeudar dos asignaturas "previas", y no tres como sucede en las escuelas provinciales. Estas dos últimas características actúan en conjunto como una doble vía de legitimación. Por un lado, colaboran con la legitimación de estas instituciones como más exigentes y de calidad educativa superior. Por otro, con la legitimación de sus egresados como triunfadores que consiguieron vencer obstáculos y exigencias que son mayores en estas escuelas que en las demás.

En cuarto lugar, en estas escuelas se cuenta con mayor libertad para la innovación pedagógica. Como señalaba Juliana:

Y además la libertad que te da la autonomía universitaria, hace que se pueda ensayar estas cosas que aunque sean dispositivos simples, desde dispositivos de acompañamiento... tener plata y recursos y posibilidad de tener un taller extraprogramático, de hacer un proyecto interdisciplinario sin tener que pedir permiso (Juliana, directora, Escuela 1).

De ese modo, ella resaltaba dos de las características que entendía como distintivas de esta institución: por un lado, la autonomía que permite la innovación y experimentación y, por otro, la posesión de recursos, como ya se ha desarrollado. En ese sentido, se trata de una escuela que sostiene un "dis- 
curso de sî" (Martínez, Villa, \& Seoane, 2009, p. 50) en el cual se proyecta como colegio de "vanguardia pedagógica". Se consideran a sí mismos, junto a las otras escuelas de pregrado, como parte de una "elite pedagógica", en tanto pueden experimentar ${ }^{3}$ e incluso transferir experiencias exitosas a escuelas provinciales que cuentan con menos recursos y, por ende, con menos capacidad para experimentar e innovar. Cabe agregar que la Escuela 1 está atravesada por la experimentación pedagógica en tanto nace ligada a las ideas de Víctor Mercante, exponente del positivismo argentino, y a la sección pedagógica de la Universidad de La Plata.

Desde la Escuela 2, la jefa del Departamento de Letras subrayaba la superioridad pedagógica de las escuelas de pregrado:

Son colegios muy elegidos. Lo importante es tener la oportunidad, porque son pocos los cupos. A veces traen cosas de otras escuelas que son conceptos erróneos. Vienen con "oración unimembre": esa no es la perspectiva que usamos acá. Están desactualizadas las otras escuelas (Docente 7, Escuela 2).

Asimismo, estas escuelas se distinguen de "las otras" porque en ellas rige el "sistema preuniversitario", constituido por la existencia de materias optativas en el último año; por la posibilidad de realizar pasantías en la universidad y por el régimen de asistencias computadas por materia y no por día de clases:

Existen articulaciones que nosotros les hacemos hacer a los chicos con pasantías académicas en la facultad, todo el tema de una currícula flexible donde pueden elegir materias y talleres, esa posibilidad de elección, el preuniversitario con esas faltas por materia... (Nadia, Directora saliente, Escuela 1).

Además del entrenamiento en la posibilidad de armar el propio recorrido de asignaturas a cursar, la existencia de optatividades habilita una "sociabilidad ampliada" al promover la mezcla de estudiantes que, perteneciendo a distintas divisiones o cursos, escogen las mismas materias optativas según sus intereses. En el énfasis colocado en la posibilidad de que escojan sus

3 Dicha libertad para experimentar se materializa en la existencia de asignaturas optativas, en el criterio del cómputo de inasistencias por materia y no por día, en la posibilidad de realizar pasantías en la universidad, en la implementación de un sistema de materias previas por promoción en la escuela 1, en el trabajo interdisciplinar (asignaturas que combinan la Literatura y la Historia o la Geometría y el Arte), o en la creación de un bloque académico entre primero y segundo año en la Escuela 3. 
propios trayectos reaparece la figura del estudiante autónomo como aquel "tipo de estudiante ideal".

Como indica Aguilar (2011) al delimitar el concepto de elite, se trata de quienes han sido elegidos y llamados a ejercer una influencia mayor a la del resto de los individuos en una comunidad. El autor retoma los estudios clásicos de los denominados teóricos de las elites: Pareto, Mosca y Michels. Entre los actores consultados prima una narrativa que sostiene que quienes forman parte de estas escuelas constituyen una minoría que tiene la función de direccionar a las demás escuelas. Se trataría de un grupo privilegiado desde el punto de vista de su capacidad de incidir en la educación al exportar experiencias exitosas.

Finalmente, otra característica distintiva señalada por los actores de estas escuelas es el peso que en ellas cobra la dinámica disciplinar o departamental. En ese sentido, las "culturas departamentales" se superponen a las "culturas escolares" en ambas instituciones.

Para concluir, Obiols \& Di Segni (1993) designan como "escuelas promedio" a aquellas que atienden mayoritariamente a hijos de padres con estudios secundarios o terciarios y cuyos docentes cuentan por lo general con título terciario no universitario. En el caso de estas escuelas, se trata de estudiantes cuyos progenitores cuentan con nivel de estudios universitario completo o incompleto en un $60 \%$ en la escuela 1 y en un $80 \%$ en la Escuela 2 y cuyos docentes se han formado en el circuito universitario. Es decir que conforman un fragmento educativo diferenciado atravesado por la experiencia universitaria.

\section{Jerarquías dentro del fragmento: distancias entre Escuela 1, Escuela 2 y Escuela 3}

A los procesos de producción de jerarquías ya analizados se superpone otro: el que se produce al interior mismo del grupo de escuelas de pregrado.

Los miembros de la Escuela 1 y 2 subrayan que el tamaño menor de estos establecimientos los constituye en "un espacio más familiar" que aquel que ofrece la Escuela 3. En ese sentido, la directora de la Escuela 2 valoraba positivamente el sentimiento de pertenencia a la institución:

Hay una tradición bastante visible en la conformación de la matrícula del colegio que tiene que ver con que vienen hijos de egresados de la escuela. 
Es una escuela que genera un fuerte sentido de pertenencia por su biografía institucional, por su historia, por las redes (de afecto, de convivencia) que se generan (Sonia, Directora, Escuela 2).

De este modo, ella señalaba a un mecanismo que evidencia cierto "acaparamiento de oportunidades" (Tilly, 2000) en relación con la reiteración de hijos de egresados en la matrícula del colegio e indicaba, asimismo, que los padres escogen esta escuela teniendo en cuenta no únicamente la calidad educativa, sino aquello que implica como institución que habilita la sociabilidad y las "redes" en cierto sector social de la ciudad. Por otra parte, la reiteración de apellidos ilustra el peso de las relaciones informales en virtud de la baja institucionalización de los circuitos de acceso a la elite en nuestro país (Tiramonti \& Ziegler, 2008). Si bien el sorteo anula la acción directa de las redes informales, éstas se activan indirectamente, a partir de la circulación de información.

La especialidad constituye, por otra parte, el elemento que más claramente diferencia a la Escuela 2, de modalidad artística, de las otras que componen el grupo de establecimientos de pregrado. En palabras de la flamante directora de la Escuela 1:

La Escuela 2 tiene su identidad definida artística. Entonces la Escuela 2 es la música, es la plástica, son las exposiciones, los violines, y la Escuela 3 es la escuela de la universidad, histórica. Nosotros siempre quedamos ahí en el medio; me parece que está emergiendo una identidad particular en la Escuela 1 , que tiene que ver con ser la escuela más pública y más popular si se quiere... (Juliana, Directora, Escuela 1).

Hay en sus palabras un juego entre la autopercepción y la heteropercepción: en la medida en que se distancia de los otros se define a sí misma. En su visión se trata, en comparación con las otras dos secundarias, de la más similar a las provinciales en cuanto a la composición de su matrícula, pero a su vez se distingue de éstas por "ofrecer una variedad distinta" en relación con la enseñanza.

Las nociones sobre los sectores sociales atendidos constituyen otro punto de distancia, en tanto se considera que la escuela artística atiende un porcentaje menor de sectores desfavorecidos en comparación con la Escuela 1:

Hace que la universidad tenga otro lugar en la comunidad, permite que sea una posibilidad para quienes antes no lo era. En la escuela 1 hay demanda de 
sectores vulnerables e inmigrantes. En la Escuela 2 no tanto, son clases medias profesionales (Docente 5, Escuela 2).

Para concluir, la "cultura escolar" (Viñao Frago, 2002) en las instituciones abordadas está atravesada por un rasgo común que refiere a una tradición selectiva que algunos de sus miembros reivindican desde visiones meritocráticas y otros impugnan desde visiones igualitaristas de la educación. Asimismo, la representación de una identidad colectiva no supone sino ficticiamente la homogeneización interna de todo el grupo. Los imaginarios que se construyen sobre los otros disimulan las diferencias internas. Al interior de este grupo de escuelas, las Escuelas 1 y 2 presentarían un "espacio más familiar" y, entre ellas, la Escuela 2 presenta la característica de que los estudiantes eligen permanecer en el establecimiento en tiempo extraescolar. El aspecto que distingue a la Escuela 2 de las demás es la especialidad artística. Con respecto a las nociones sobre los sectores sociales atendidos, la Escuela 1 considera que atiende a sectores heterogéneos mientras que los docentes señalan que la escuela artística concentra grupos favorecidos. Por último, desde la Escuela 2 se sostiene un "discurso de sî" que indica que es allí donde la oferta es más completa y donde se forman los sujetos más creativos.

\section{Jerarquías superpuestas o la elite dentro de la elite: la escuela artística por dentro}

Otro proceso de diferenciación que se superpone con los anteriores se produce entre las dos especialidades que ofrece la Escuela 2. Como hemos indicado, esta escuela Secundaria de modalidad artística habilita dos tipos de bachillerato: Bachiller en Música y Bachiller en Artes Visuales ${ }^{4}$.

$\mathrm{Al}$ indagar sobre las visiones de los actores en torno a las diferencias entre las especialidades, varios referían a que "Discursos Visuales" constituye una orientación más "fácil" que "Discursos Musicales": "Discursos Visua-

4 A su vez, cada orientación presenta tres tipos de especialidad: Bachiller en Música (Guitarra/ Piano/ Violín/ Flauta/ Violoncello/ Saxofón/ Contrabajo): Especialidad Producción Instrumental, Composición Experimental o Artística Sociocomunitaria; Bachiller en Artes Visuales (Pintura/ Grabado/ Escultura): Especialidad Producción Proyectual, Composición Experimental o Artística Sociocomunitaria. 
les es más fácil. Es algo que tiene que ver con la exigencia. En más fácil entrar con ese asunto del dibujo" (Docente $n^{\circ} 32$, escuela 2 ).

Desde estas miradas, la especialidad música implica un grado de disciplinamiento mayor en comparación con Discursos Visuales. Otro de los aspectos que los docentes indicaban como distintivo de Discursos Visuales se relacionaba con el trabajo grupal que esta especialidad ofrece, en contraste con el trabajo individual propio de quienes deben aprender a dominar un instrumento:

Siendo estricto, música implica mucho más trabajo. En Discursos Visuales sociabilizan mucho, trabajan con compañeros, hacen trabajos manuales. Es música trabajás de forma solitaria, mucho más tiempo. Es como un deporte de alto rendimiento (Docente $\mathrm{n}^{\circ} 22$, escuela 2).

Otros argumentos referían a que en el caso de música era necesario, o bien haber estudiado un instrumento previamente, o bien tener "talento" para iniciarse en ello. Entretanto, en Discursos Visuales no era necesario conocer técnicas de dibujo de antemano, sino que cualquier persona estaría potencialmente capacitada o podría desarrollar esas habilidades durante la escolarización: "Es más general, para música tienen que tener real vocación para la música. Todo el mundo tiene una veta para el arte manual" (Docente $\mathrm{n}^{\circ} 28$, escuela 2 ).

De ese modo se produce una jerarquización de los estudiantes de música de la escuela y de los músicos en general, pensándolos como provistos de un talento especial, formando "la elite de la elite". Desde este enfoque se concibe a los artistas como héroes solitarios y disciplinados.

En consonancia con lo antedicho, al consultar a los docentes en qué orientación encuentran a los mejores estudiantes, se observa cierta coincidencia en que las/os estudiantes de música son mejores: "Música es más exigente. Es otro target, lo de Discursos Visuales son más vagos" (Docente $n^{\circ} 32$, escuela 2).

En conclusión, los procesos de autolegitimación por distanciamiento se multiplican, se solapan y se superponen produciendo jerarquizaciones incluso al interior mismo de las instituciones, a partir de negociaciones de fronteras porosas. Específicamente en el caso de la Escuela 2 se habilita un juego de hetero y autopercepciones del cual resulta que una de las especialidades es considerada superior a partir de que se le atribuye mayor dificultad, exigencia y rigurosidad. 


\section{Discusión y conclusiones}

En este trabajo se analizaron los procesos de fabricación de jerarquías y de afirmación de distancias con respecto a "los otros" presentes en un grupo de escuelas secundarias dependientes de Universidades Nacionales en Argentina. Como se ha mostrado, estas instituciones trabajan activamente en la legitimación mediante el distanciamiento y la subaternización de sus "otros" a partir de la movilización de criterios de clasificación simbólica. Así, el reforzamiento de las jerarquías respecto de los otros afianza su posicionamiento en un lugar de superioridad dando lugar a procesos de autolegitimación por distanciamiento. Se analizó, por otra parte, cómo dichos procesos, lejos de distribuirse según la dicotomía arriba- abajo, se multiplican y yuxtaponen incluso al interior mismo del grupo de escuelas estudiadas y también dentro de las instituciones. En ese sentido, se mostró que no se trata de un cuerpo monolítico perfectamente integrado ni de un grupo que ejerce superioridad sobre las demás escuelas articuladamente sino de un campo en disputa en que en el que se invoca un "espíritu de cuerpo" (Bourdieu, 2013) siempre que es preciso tomar distancia de las "otras escuelas" provinciales y privadas en pos de la consolidación de su lugar de elite en el sector estatal de la educación secundaria.

Como ha indicado Weber (1969), la desigualdad necesita ser legitimada y esto implica que quienes ocupan un lugar de superioridad trabajen en pos de tornar las desigualdades aceptables. El cúmulo de categorías antes desarrolladas permite concebir el trabajo llevado adelante por parte de los actores de estas escuelas en pos de diferenciarse de los "otros" y ubicarse en un lugar de elite: los otros pueden ser escuelas privadas, provinciales u otras escuelas o especialidades dentro de las universitarias.

\section{Bibliografía}

Aguilar, O. (2011). Dinero, educación y moral: el cierre social de la elite tradicional chilena. En Joinant, A. y Guell, P. (Eds.), Notables, tecnócratas y mandarines. Elementos de la sociología de las elites en Chile (1990-2010). Chile: Salesianos impresores.

Bourdieu, P. (2013). La nobleza de Estado. Buenos Aires: Siglo XXI Editores. 
Cohen, G.A. (1996). ¿Igualdad de qué? Sobre el bienestar, los bienes y las capacidades. En Nussbaum, M. y Sen, A. (comp), La calidad de vida. México DF: Fondo de Cultura Económica.

Crozier, G. et al. (2008). White middle class parents, identities, educational choice and the urban comprehensive school: dilemmas, ambivalence and moral ambiguity. British Journal of Sociology of Education, 29(3), 261-272.

Eisenhardt, K.M. (1989). Building Theories from Case Study Research. Academy of Management Review (14), 4.

Elias, N. (1998). Ensayo teórico sobre las relaciones entre establecidos y marginados. En La civilización de los padres y otros ensayos. Bogotá: Editorial Norma.

Kessler, G. (2002). La experiencia escolar fragmentada. Estudiantes y docentes en la escuela media en Buenos Aires. Buenos Aires: UNESCO, I.I.P.E.

Latour, B. (2008). Reensamblar lo social. Una introducción a la teoría del actorred. Buenos Aires: Manantial.

Martínez, M. E., Villa, A. \& Seoane, V. (Coord.) (2009). Jóvenes, elección escolar y distinción social. Investigaciones en Argentina y Brasil. Buenos Aires: Prometeo Libros.

Núñez, P. \& Litichever, L. (2015). Radiografía de la experiencia escolar. Ser joven en la escuela. Grupo Editor Universitario, CLACSO.

Obiols, G. \& Di Segni de Obiols, S. (1993). Adolescencia, posmodernidad y escuela secundaria. La crisis de la enseñanza media. Buenos Aires: Kapeluz.

Reygadas, L. (2008). Distinción y reciprocidad. Notas para una antropología de la equidad. Nueva antropología, 21(69), 9-31.

Saraví, G. (2015). Juventudes fragmentadas: socialización, clase y cultura en la construcción de la desigualdad. México: FLACSO.

Stich, A. E. \& Colyar, J. E. (2013). Thinking relationally about studying 'up'. British Journal of Sociology of Education, https://doi.org/10.1080/0142569 2.2013.843954

Stake, R. (1995). Investigación con estudios de caso. Madrid: Ediciones Morata.

Tenti Fanfani, E. (2011). La escuela y la cuestión social. Ensayos de sociología de la educación. Buenos Aires: Siglo XXI Editores.

Tilly, C. (2000) La desigualdad persistente. Buenos Aires: Manantial.

Tiramonti, G. (Comp.) (2004). La trama de la desigualdad educativa. Mutaciones recientes en la escuela media. Buenos Aires: Manantial.

Tiramonti, G. \& Ziegler, S. (Comp.) (2008). La educación de las elites. Aspiraciones, estrategias y oportunidades. Buenos Aires: Paidós. 
Viñao Frago, A. (2002). Sistemas educativos, culturas escolares y reformas. Madrid: Morata.

Walzer, M. (1993). Las esferas de la justicia. México DF: Fondo de Cultura Económica.

Weber, M. (1969). Economía y sociedad. México: Fondo de Cultura Económica.

\section{Documentos y fuentes}

Universidad Nacional de La Plata (2001). Nuestro Liceo. La Plata: UNLP.

Fecha de recepción: 2017/11/10; fecha de aceptación: 2018/02/19;

fecha de publicación: 2018/03/0I 